\title{
Grid-Based Environment Estimation Using Evidential Mapping and Particle Tracking
}

\author{
Sascha Steyer, Georg Tanzmeister, and Dirk Wollherr
}

\begin{abstract}
Modeling and estimating the current local environment by processing sensor measurement data is essential for intelligent vehicles. Static obstacles, dynamic objects, and free space have to be appropriately represented, classified, and filtered. Occupancy grids, known for mapping static environments, provide a common low-level representation using occupancy probabilities with an implicit data association through the discrete grid structure. Extending this idea toward dynamic environments with moving objects requires a static/dynamic classification of measured occupancy and a tracking of the dynamic state of grid cells. In this work, we propose a new dynamic grid mapping approach. An evidential representation using the Dempster-Shafer framework is used to model hypotheses for static occupancy, dynamic occupancy, free space, and their combined hypotheses. These hypotheses are consistently estimated and accumulated in a dynamic grid map by an adapted evidential filtering, allowing one to distinguish static and dynamic occupancy. The evidential grid mapping is combined with a low-level particle filter tracking that is used to estimate cell velocity distributions and predict dynamic occupancy of the grid map. Static occupancy is directly modeled in the grid map without requiring particles, increasing efficiency and improving the static/dynamic classification due to the persistent map accumulation. Experimental results with real sensor data show the effectiveness of the proposed approach in challenging scenarios with occlusions and dense traffic.
\end{abstract}

Index Terms-Autonomous vehicles, dynamic occupancy grids, environment perception, mapping, object detection, tracking.

\section{INTRODUCTION}

$\mathbf{E}$ NVIRONMENT perception enables robots to interpret the current state of their surroundings and interact with them. Estimating the local dynamic environment of autonomous vehicles is a major challenge due to the high scenario complexity, sensor limitations, and high demands on accuracy and robustness. A consistent model is required that represents static obstacles, moving objects, and free space. This model has to be estimated by processing the measurement data of different sensors, requiring data fusion, temporal filtering, and classification regarding the modeled states.

A common approach for estimating objects is a sensorindividual data processing in which objects are extracted by specific features, temporally filtered, and eventually fused on that high-level representation with the object tracks of other sensors [1]-[3]. Occupancy grids [4], [5], in contrast, provide a robust low-level representation using occupancy probabilities of grid cells to model both the occupancy of static obstacles

Manuscript received December 23, 2017; revised March 22, 2018; accepted May 13, 2018. This work was supported by the BMW Group, Germany.

S. Steyer and G. Tanzmeister are with the BMW Group, Munich, Germany. E-mail: sascha.steyer@bmw.de, georg.tanzmeister@bmw.de

D. Wollherr is with the Institute of Automatic Control Engineering (LSR), Technical University of Munich, Munich, Germany. E-mail: dw@tum. de and the free space. The discrete grid structure implicitly performs the association for both the fusion of different sensor data and the mapping as a temporal accumulation without requiring object assumptions. Within the last few years, the idea of occupancy grid mapping has been extended toward dynamic environments with moving objects by additionally estimating the dynamic state of the grid. Recent approaches use particle filters to estimate cell velocities and distinguish between static obstacles and dynamic objects. That resulting dynamic grid representation can also be used as input to a high-level object tracking, taking advantage of the low-level grid cell fusion, static/dynamic classification, and velocity estimation.

In our previous work [6], we presented such a grid-based object tracking approach. As input, we used the dynamic occupancy grid estimation by Tanzmeister and Wollherr [7]. They proposed a grid-based particle tracking for estimating the dynamic state of the occupied environment. Each particle corresponds to a hypothesis of occupancy at a particular position with a particular velocity. Hence, the particle population represents a filtered estimation of occupancy and velocity distributions, but it is a short-term estimation since particles are only distributed in areas of currently measured occupancy. The particles are used to classify measured occupancy into static and dynamic, which are modeled as individual hypotheses using a Dempster-Shafer evidential representation. This output of classified measurement data is additionally accumulated in a grid map, resulting in a persistent estimation of the static environment. That tracking and mapping approach shows promising results, however, a major drawback is that no information of the accumulated map is used for the particle tracking. Thus, particle destruction due to temporary occlusion leads to information loss. This partly results in incorrect dynamic classification of previously correctly accumulated static occupancy, which in turn increases falsely extracted objects.

This work focuses on improving the dynamic estimation and classification on the grid cell-level, also motivated to enhance the robustness of the object tracking approach based on it. As a main difference to [7], we use a modified combination of the particle filter tracking and the evidential grid mapping. Input to the particle tracking is the classified and filtered evidential grid map rather than the measurement data directly. Hence, the persistent static occupancy estimation of the map is used as input to the short-term particle tracking to avoid an incorrect initialization of dynamic particles in grid cells with previously accumulated static occupancy.

This new architecture also fundamentally changes the filtering process. The evidential dynamic grid map forms the main component where the predicted state is updated by the mea- 
surement data. Static occupancy is directly filtered by this map without requiring static particles, i. e., particles solely represent dynamic occupancy, which significantly reduces the number of required particles. Dynamic occupancy is also equally updated by the map instead of using individual particle survival probabilities. The main task of the particle tracking is thus to estimate cell velocity distributions, which in turn are used to predict dynamic occupancy evidence of the map.

The evidential dynamic grid map represents hypotheses of static occupancy, dynamic occupancy, and free space, as well as the hypotheses supersets of unclassified occupancy, passable area that may be temporarily occupied, and an unknown state. In extension to the evidential representation of [7], the hypothesis of passable area is introduced to model the relation between measured free space and dynamic occupancy. This allows for a consistent prediction, an implicit classification of dynamic occupancy, and a differentiation between previously measured free space that may be temporarily occupied and currently measured free space.

Overall, we propose a new grid-based estimation of dynamic environments that combines the benefits of a multi-hypotheses evidential representation, a short-term particle tracking of the dynamic state, a persistent grid mapping of the static environment, and a consistent filtering of all modeled hypotheses. Regarding the application in autonomous vehicles, this ultimately improves the collision avoidance with static obstacles and, especially by decreasing falsely extracted moving objects, the dynamic maneuver planning with surrounding traffic participants, while at the same time the computational effort of the real-time system is significantly reduced.

The paper is structured as follows: Sec. II presents related work and Sec. III introduces the evidential dynamic grid map and particle representation. The prediction and update of the dynamic grid map and particle population are discussed in Sec. IV. Experimental results with real sensor data including a comparison to the original approach are shown in Sec. V.

\section{RELATED WORK}

The idea of occupancy grids as described in the introduction is widely used in various mapping applications. Dynamic environments with moving objects require an additional estimation of the dynamic state to detect and predict movements as part of the temporal filtering. Various approaches exist for the dynamic estimation on the grid cell-level, typically referred to as dynamic occupancy grids.

A simple approach to distinguish static and dynamic grid cells is to determine inconsistencies between the accumulated map and new measurements [8], [9]. For example, a cell that has accumulated a high free space probability in the map but has a high occupancy probability in the current measurement has to be the result of a moving object, i.e., a dynamic cell. Equivalently, conflict masses are analyzed in [10], [11] using the Dempster-Shafer evidential framework, which is further extended in [12] using prior map knowledge, and a modified evidential combination and accumulation. In addition, object hypotheses can be extracted and tracked using distance-based clustering of those detected moving parts, e. g.
[11], [13]-[16]. However, a static/dynamic classification based solely on analyzing inconsistencies or conflicts is error-prone to measurement inaccuracies of ranging or odometry sensors. Even though results of an object tracking can be used to improve this classification, no robust cell-individual velocity estimation is achieved this way.

In [17], [18] the Bayesian occupancy filter (BOF) is introduced, an approach that recursively estimates the dynamic state of an occupancy grid through discrete cell velocities modeled as neighborhood cell transition histograms. But an accurate and wide range cell velocity estimation using these transition histograms of each cell requires enormous computing/memory consumptions and the discretization also leads to aliasing problems. However, this approach has been extended in various interesting approaches - a detailed review of the BOF including multiple extensions is given in [19].

Most promising approaches use particle filters to estimate the dynamic state of the occupancy grid, partly referred to as Sequential Monte Carlo BOF (SMC-BOF). Originally proposed by Danescu et al. [20], [21], each particle represents a point mass of occupancy with continuous position and velocity that moves freely in the entire grid structure. The number of particles in a cell is adapted proportionally to the cell occupancy value. The sampling, weighting, and resampling of the particles results in a robust filtering of both the occupancy probabilities and the multimodal velocity distribution for each grid cell. A cell is classified as either static or dynamic by evaluating the velocity variance of all particles in that cell.

The grid-based particle filtering idea of [20] has been adapted by Tanzmeister et al. [7], [22] toward an evidential representation using the Dempster-Shafer theory and an additional mapping, referred to as evidential grid-based tracking and mapping (GTAM) [7]. In addition to the division of occupancy probabilities into separate evidence for occupancy and free space, as in [10], the occupancy hypothesis is subdivided into the two hypotheses of static and dynamic occupancy, which are then estimated continuously as opposed to the discrete classification of [20]. In addition to an initial uniform velocity distribution of new particles, pure static particles with zero velocity are sampled that improve convergence of static occupancy. The number of particles and the occupancy evidence in a cell are defined by the measured occupancy belief, while additional particle survival probabilities enable short persistence in occluded areas. Moreover, the output of the particle filtering is temporally accumulated, allowing for full persistence of the static environment in a grid map representation without keeping all particles. However, information of the accumulated map is not considered in the particle filtering and dynamic evidence masses are not accumulated, i. e., multiple measurements do not increase the dynamic masses.

Nègre et al. introduced the idea of hybrid sampling BOF (HSBOF) [23] as an adaptation of [18] and [20] toward a decomposition of static and dynamic occupancy. Static occupancy is represented in a typical occupancy grid, whereas dynamic occupancy is modeled by particles. This means that the particle population only represents the dynamic environment rather than all occupied areas, which significantly reduces the number of required particles. The same authors improved this 
idea in [24] by introducing formal states of static occupancy, dynamic occupancy, free space, and unknown areas in the filtering process, resulting in a more accurate velocity estimation and compact algorithm definition. However, in contrast to the evidential representation of [7], no dependencies of the individual hypotheses and their supersets are modeled by these states. Furthermore, the temporal filtering and the combination of the states are not described in detail.

Nuss et al. [25] also presented an SMC-BOF variant based on [20]-[23]. They focused on fusing laser and radar data, and improving the particle velocity estimation using radar Doppler velocity measurements, as similarly described in [7]. An extension and reformulation of Nuss et al. [26] models the grid-based dynamic estimation as a random finite set (RFS) problem using a combination of a Bernoulli filter and a PHD filter. Since that approach is not really real-time capable, they also proposed an approximation using Dempster-Shafer evidence masses that is similar to [7], restricting particles to areas of currently visible occupancy. However, static and dynamic occupancy are not individually estimated. Since occupancy is only filtered by the particles without an additional grid map accumulation, occlusion also leads to particle destruction and information loss of static occupancy.

In conclusion, the different variants of the particle-based dynamic occupancy grids already show promising results. Nevertheless, these approaches do not fully combine the benefits of a multi-hypotheses evidential representation, a persistent grid mapping accumulation of the static environment together with a consistent filtering of all evidence masses, and an efficient particle tracking that is restricted to areas of dynamic occupancy. Furthermore, none of these particle-based approaches models a relation between dynamic occupancy and free space. For example, no information of previously measured free space is used as an indication that the newly measured occupancy of that same grid cell is more likely of a dynamic object than a static obstacle. In the following, these different aspects are addressed to achieve a more robust and efficient dynamic grid mapping approach that, combined with grid-based object tracking [6], improves the collision avoidance and maneuver planning of autonomous driving applications.

\section{Evidential Map and Particle Representation}

In this section, the selected evidential map representation including the set of possible hypotheses, the basic combination principle of belief masses, and the low-level dynamic state estimation using a particle filter are presented.

\section{A. Map Representation with Evidential Frame of Discernment}

The occupancy grid map of the local environment is estimated using the Dempster-Shafer theory of evidence (DST) [27], [28], which can be seen as a generalization of the Bayesian theory [29]. The frame of discernment

$$
\begin{aligned}
\Theta & =\{F, S, D\}, \\
2^{\Theta} & =\{\{F\},\{S\},\{D\},\{S, D\},\{F, D\}, \Theta, \emptyset,\{S, F\}\}
\end{aligned}
$$

with the individual hypotheses
$\{F\}$ passable area, currently free

$\{S\}$ not passable area, statically occupied

$\{D\}$ passable area, currently dynamically occupied

$\{S, D\}$ currently occupied (temporarily or permanently)

$\{F, D\}$ passable area (free or dynamically occupied)

$\{\Theta\}$ unknown state, i. e., either free or occupied

is used similarly as proposed in [7]. The set $\{S, F\}$ is also not used in this work as it is conflicting by definition. But, as a difference, we consider the set $\{F, D\}$ of passable area. This hypothesis is always conflicting for a single measurement, but is a reasonable hypothesis in the temporal filtering since $\{F\}$ and $\{D\}$ are interchangeable over time. For example, a cell that has been observed as free space $\{F\}$ may be occupied one time step later by a moving object, modeled as dynamic occupancy $\{D\}$, which moves across that cell. In sum, the superset $\{S, D\}$ primarily models measured occupancy at a single time that cannot directly be classified as $\{S\}$ or $\{D\}$, whereas $\{F, D\}$ models the uncertain state of measured free space predicted to a different time.

In the DST framework, a basic belief mass $m(\cdot) \in(0,1)$ is assigned to each hypothesis of the power set $2^{\Theta}$ such that the sum of all basic belief masses equals 1 . The sum of all subsets $\tilde{\theta} \subseteq \theta$ of a hypothesis $\theta \subseteq \Theta$ is denoted as a belief

$$
\operatorname{bel}(\theta)=\sum_{\tilde{\theta} \subseteq \theta} m(\tilde{\theta}) \leq p(\theta)
$$

and is a lower bound of the probability $p(\theta)$. Using the frame of discernment as defined in (1), the occupancy belief

$$
\operatorname{bel}(O)=\operatorname{bel}(S D)=m(S)+m(D)+m(S D)
$$

is subdivided into the three possible basic belief masses of static occupancy $m(S)$, dynamic occupancy $m(D)$, and unclassified occupancy $m(S D)$. In this work, the argument of the belief mass, i.e., the corresponding hypothesis set, is abbreviated without brackets or comma for better readability. The basic belief mass of the unknown state

$$
m(\Theta)=1-\sum_{\theta \subset \Theta} m(\theta)
$$

can always be calculated as the remaining mass and is not explicitly stated in the following.

In sum, the accumulated evidential dynamic occupancy grid map at time $t$ is described by

$$
\mathcal{M}_{t}=\left[m\left(S_{t}\right), m\left(D_{t}\right), m\left(S D_{t}\right), m\left(F_{t}\right), m\left(F D_{t}\right)\right]^{\top} \text {. }
$$

These evidence masses are defined for all cells $c \in \mathcal{G}$ of the grid structure $\mathcal{G}$, where the cell index $c$ is omitted for better readability when irrelevant. A visualization of the dynamic grid map is shown in Fig. 1a. The evidence color coding is selected as

$$
\mathrm{RGB}=\left(1-\sum_{\theta \cap\{S\}=\emptyset} m(\theta), 1-\sum_{\theta \cap\{F\}=\emptyset} m(\theta), 1-\sum_{\theta \cap\{D\}=\emptyset} m(\theta)\right)
$$

for all hypotheses $\theta \subset \Theta$. This means that static occupancy results in red $(R)$, free space in green $(G)$, dynamic occupancy in blue (B), unclassified occupancy in pink $(\mathrm{R}+\mathrm{B})$, passable area in cyan $(\mathrm{G}+\mathrm{B})$, and an unknown state in white. 


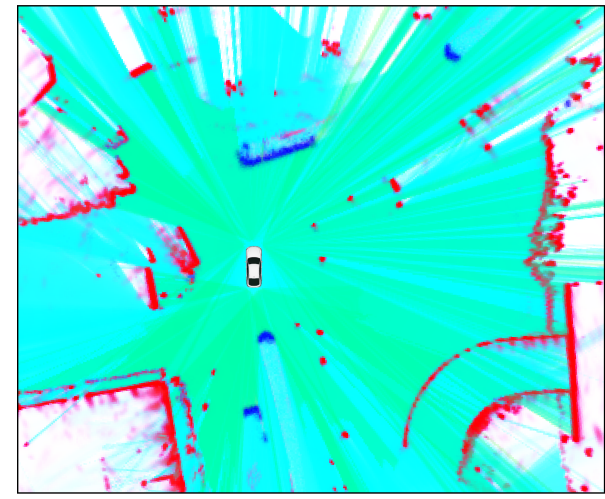

(a)

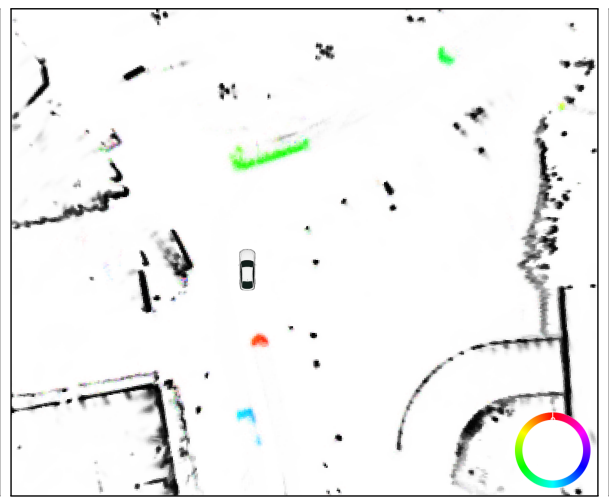

(b)

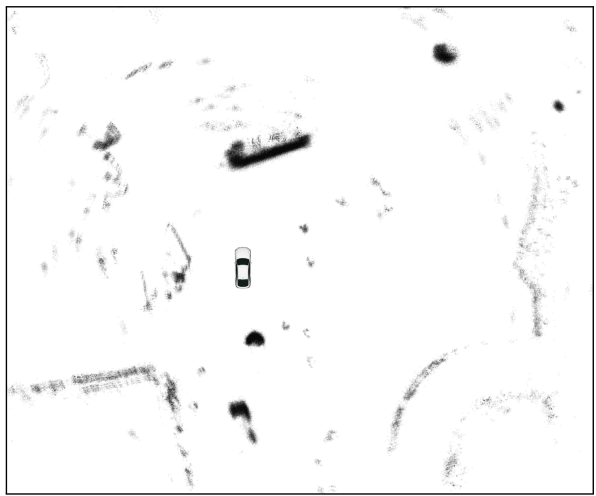

(c)

Fig. 1. Grid and particle visualization. (a) Evidential dynamic occupancy grid map using (6). (b) Velocity orientation grid using (12). (c) Particle population.

\section{B. Basic Combination Principle of Belief Masses}

Belief masses of different grids $\mathcal{M}_{i}$, all representing the same time $t$, are combined in the DST framework by considering the intersections of the hypotheses sets $\Theta_{i}=\left\{F_{i}, S_{i}, D_{i}\right\}$ between the grids $\mathcal{M}_{i}$. Various rules of combination exist. The conjunctive rule of combination that forms the basic intersection is calculated as

$$
m\left(\theta \mid \mathcal{M}_{1} \oplus_{\mathrm{c}} \mathcal{M}_{2}\right)=\sum_{\alpha_{1} \cap \beta_{2}=\theta} m\left(\alpha_{1}\right) m\left(\beta_{2}\right)
$$

with $\theta \subseteq \Theta, \alpha_{1} \subseteq \Theta_{1}, \beta_{2} \subseteq \Theta_{2}, \Theta_{1} \cap \Theta_{2}=\Theta$. The evidence of the sum of intersections resulting in the empty set $\emptyset$ is represented by the conflict mass

$$
\zeta\left(\mathcal{M}_{1}, \mathcal{M}_{2}\right)=\sum_{\alpha_{1} \cap \beta_{2}=\emptyset} m\left(\alpha_{1}\right) m\left(\beta_{2}\right) .
$$

This conflict can be assigned in different ways. Most commonly, Dempster's rule of combination [28]

$$
m\left(\theta \mid \mathcal{M}_{1} \oplus_{\mathrm{d}} \mathcal{M}_{2}\right)=\frac{m\left(\theta \mid \mathcal{M}_{1} \oplus_{\mathrm{c}} \mathcal{M}_{2}\right)}{1-\zeta\left(\mathcal{M}_{1}, \mathcal{M}_{2}\right)}, \zeta<1
$$

is used, which normalizes (7) by an inverse of the conflict mass, meaning that the conflict mass is assigned proportionally to the belief masses of the intersections. The conflict can, for example, also be assigned to the unknown set $m(\Theta)$, the empty set $m(\emptyset)$ if $\Theta$ is not exhaustive, the union set $\alpha_{1} \cup \beta_{2}$, or distributed individually based on expert knowledge [30]. The latter will be used in this work to solve the prediction and measurement update with specific behavior.

\section{Particle Population}

The dynamic state of the map is estimated using a particle filter as proposed in different approaches [7], [20]-[26]. The individual particles $\chi \in \mathcal{X}_{t}$ of the particle population $\mathcal{X}_{t}$ at time $t$ represent points of occupancy. Each particle is described by a position $x_{\chi} \in \mathbb{R}^{2}$, a velocity $v_{\chi} \in \mathbb{R}^{2}$, and an occupancy evidence amount $o_{\chi} \in[0,1)$. The particle population $\mathcal{X}_{t}^{c}$ describes all particles $\chi$ associated to a grid cell $c \in \mathcal{G}$ regarding the current particle position $x_{\chi}$. The sum of all particle occupancy values in a cell $c$

$$
\sum_{\chi \in \mathcal{X}_{t}^{c}} o_{\chi}=m\left(D_{t}^{c}\right)
$$

is selected to be equal to the dynamic evidence mass $m\left(D_{t}^{c}\right)$ of that cell.

The main task of the particle filtering is a robust low-level velocity estimation and a prediction of the dynamic evidence masses by the individual particles. A cell velocity

$$
v_{c, t}=\frac{1}{m\left(D_{t}^{c}\right)} \sum_{\chi \in \mathcal{X}_{t}^{c}} o_{\chi} v_{\chi} \in \mathbb{R}^{2}
$$

is calculated as the mean particle velocity of all particles in a cell, weighted by their occupancy values that sum up to the dynamic occupancy mass. The static occupancy mass is not considered in (11) to obtain a bimodal velocity distribution, i. e., a mean velocity $v_{c, t}$ of the dynamic hypothesis and a zero velocity hypothesis represented by the static occupancy evidence. An illustration of the estimated velocity orientation is shown in Fig. 1b using a hue/saturation/value (HSV) color coding as proposed in [22]:

$$
\mathrm{HSV}=\left(\arctan \left(\frac{v_{c, t}^{y}}{v_{c, t}^{x}}\right), m\left(D_{t}^{c}\right), 1-m\left(S_{t}^{c}\right)\right) .
$$

The corresponding particle population $\mathcal{X}_{t}$ is depicted in Fig. 1c. Particles represent only hypotheses of dynamic occupancy, similarly proposed in [23]. Particles are therefore only drawn in cells with dynamic occupancy $m(D)$ or, to initialize new dynamic evidence, in cells with unclassified occupancy $m(S D)$. Static occupancy $m(S)$, however, is solely estimated by the map $\mathcal{M}_{t}$. Thus, in contrast to [7], no static particles are sampled, which significantly reduces the number of required particles. Furthermore, the dynamic mass of the particle population is consistently filtered by the map and not by particle survival probabilities.

\section{Prediction and Update of Dynamic Grid MaP}

To update the dynamic occupancy grid map with a new measurement, i. e., the temporal filtering, the map $\mathcal{M}_{t-1}$ has to be predicted to the time $t$ of the incoming measurement. As explained in the previous section, the particle population is linked to the dynamic mass and primarily performs the velocity estimation and thus the prediction part. The incoming measurement is called a scan grid $\mathcal{M}_{z, t}$, an evidential grid representation of unaccumulated measurements resulting from the fusion of multiple sensors or a single sensor. An overview 
of the proposed prediction and measurement update of the dynamic grid map and the particle population is schematically shown in Fig. 2 and exemplarily depicted in Fig. 3. These different steps are discussed in more detail in this section.

\section{A. Prediction of Dynamic Grid Map}

The predicted dynamic grid map at time $t$ is estimated in three steps. First, the dynamic evidence is predicted using the particle population resulting in a particle grid $\widehat{\mathcal{M}}_{t}$. Secondly, the previous map $\mathcal{M}_{t-1}$ is adapted to a map $\mathcal{M}_{t}^{\prime}$ that does not contain dynamic evidence and handles the prediction of free space evidence. Finally, both grids are combined, resulting in the predicted dynamic grid map

$$
\overline{\mathcal{M}}_{t}=(1-\bar{\varepsilon})\left(\widehat{\mathcal{M}}_{t} \oplus \mathcal{M}_{t}^{\prime}\right) .
$$

The temporal uncertainty of the prediction is modeled by a reduction factor $\bar{\varepsilon}$, which, however, is omitted in the following discussion for better readability.

1) Prediction of Particle Population: The previous particle population $\mathcal{X}_{t-1}$ is predicted to time $t$ using a constant velocity motion model and additive Gaussian noise, resulting in a predicted population $\hat{\mathcal{X}}_{t}$. A predicted particle dynamic evidence mass

$$
m\left(\widehat{D}_{t}^{c}\right)=\min \left(1-\varepsilon_{o}, \sum_{\chi \in \hat{\mathcal{X}}_{t}^{c}} o_{\chi}\right), \varepsilon_{o} \in(0,1)
$$

of each grid cell $c \in \mathcal{G}$ is derived by computing the sum of the occupancy values of all particles predicted to that cell regarding the particle positions $x_{\chi}$. The maximum value has to be limited to $1-\varepsilon_{o}$ to obtain valid evidence masses and avoid saturation. The predicted particle grid $\widehat{\mathcal{M}}_{t}$ consists only of the predicted dynamic evidence mass $m\left(\widehat{D}_{t}\right)$, i. e., no other evidence is derived.

2) Prediction of Previous Map Without Dynamic Evidence: The previous map without the dynamic evidence is predicted to a map $\mathcal{M}_{t}^{\prime}$ with

$$
\begin{aligned}
m\left(S_{t}^{\prime}\right) & =m\left(S_{t-1}\right), \\
m\left(S D_{t}^{\prime}\right) & =m\left(S D_{t-1}\right), \\
m\left(D_{t}^{\prime}\right) & =0, \\
m\left(F_{t}^{\prime}\right) & =0, \\
m\left(F D_{t}^{\prime}\right) & =\frac{m\left(F D_{t-1}\right)+m\left(F_{t-1}\right)}{1-m\left(D_{t-1}\right)} .
\end{aligned}
$$

Free space evidence is transferred in the prediction to the superset $\{F, D\}$ of passable area since the state $\{F\}$ is timedependent and may have changed to dynamically occupied.

Dynamic evidence that has been predicted to a cell decreases the evidence of previously accumulated passable area in that cell, as $\{D\}$ is a subset of $\{F, D\}$. However, this accumulated passable area evidence should remain after a dynamic object leaves this cell, especially when the dynamic mass predicted to that cell has not been confirmed by any measurement. This normalization is performed by the denominator in (15), which represents the reciprocal of the reduction by a dynamic mass. Note that $m\left(D_{t-1}\right)$ is not transferred to $m\left(F D_{t}^{\prime}\right)$, as, in contrast to the measured free space $m\left(F_{t-1}\right)$,

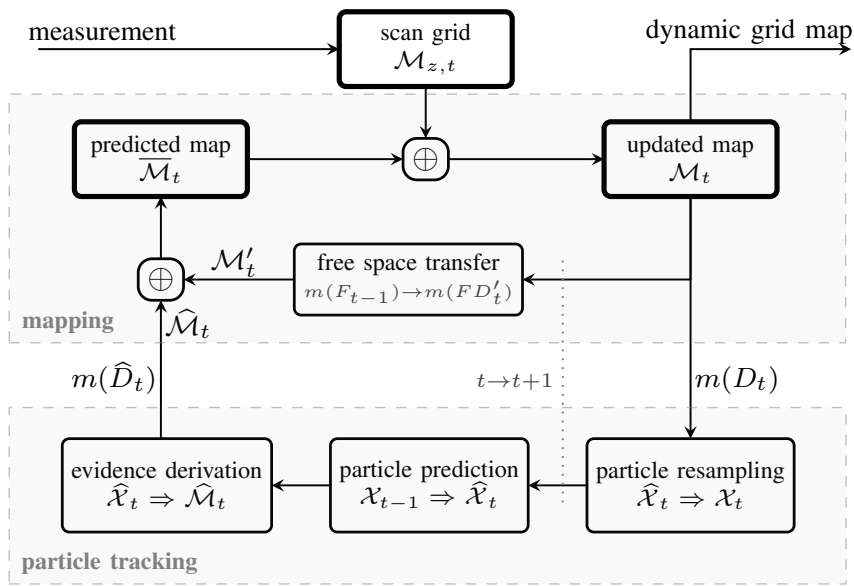

Fig. 2. Overview of the proposed mapping and particle tracking approach

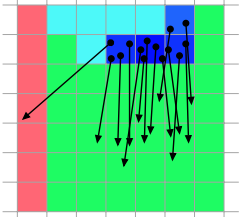

(a) $\mathcal{M}_{t-1}$

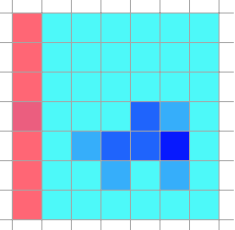

(d) $\overline{\mathcal{M}}_{t}$

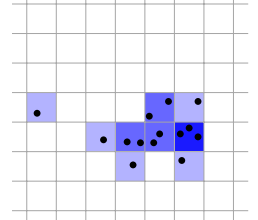

(b) $\widehat{\mathcal{M}}_{t}$

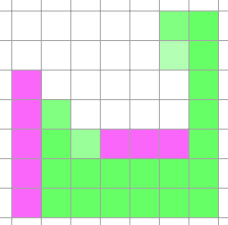

(e) $\mathcal{M}_{z, t}$

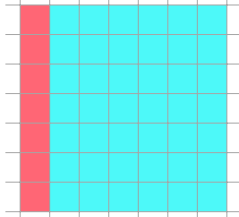

(c) $\mathcal{M}_{t}^{\prime}$

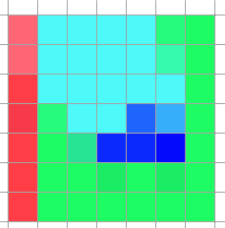

(f) $\mathcal{M}_{t}$
Fig. 3. Illustration of map prediction using the particle population and update with the new measurement. (a) Old dynamic grid map and particle population. (b) Predicted particle population and derived dynamic evidence masses. (c) Prediction without dynamic evidence. (d) Combined predicted map. (e) Scan grid of new measurement. (f) New updated dynamic grid map.

the dynamic mass is not directly measured and is derived by a prediction. Hence, that implication would be critical for wrongly predicted dynamic masses.

3) Resulting Predicted Dynamic Grid Map: The combination (13) of the predicted particle grid with the adapted previous map causes one conflict

$$
\zeta\left(\widehat{\mathcal{M}}_{t}, \mathcal{M}_{t}^{\prime}\right)=m\left(S_{t}^{\prime}\right) m\left(\widehat{D}_{t}\right) \equiv \bar{\zeta},
$$

as defined in (8). This static/dynamic conflict mass is assigned to the predicted static mass

$$
m\left(\bar{S}_{t}\right)=m\left(S \mid \widehat{\mathcal{M}}_{t} \oplus_{\mathrm{c}} \mathcal{M}_{t}^{\prime}\right)+\bar{\zeta}=m\left(S_{t-1}\right),
$$

meaning that the accumulated static evidence dominates the predicted particle dynamic evidence in the case of a conflict, i. e., $m(S)$ remains unchanged in the prediction. All remaining predicted hypotheses are combined using (7) without additional conflict assignment:

$$
m\left(\bar{\theta}_{t}\right)=m\left(\theta \mid \widehat{\mathcal{M}}_{t} \oplus_{\mathrm{c}} \mathcal{M}_{t}^{\prime}\right), \quad \theta \subset \Theta \backslash\{S\} .
$$




\section{B. Update of Predicted Dynamic Grid Map with Scan Grid}

The predicted map $\overline{\mathcal{M}}_{t}$ is updated with the two measured hypotheses of unclassified occupancy $m\left(S D_{z, t}\right)$ and free space $m\left(F_{z, t}\right)$ that form the scan grid $\mathcal{M}_{z, t}$. The combination of $\overline{\mathcal{M}}_{t}$ and $\mathcal{M}_{z, t}$ results in the updated map

$$
\mathcal{M}_{t}=\overline{\mathcal{M}}_{t} \oplus \mathcal{M}_{z, t}
$$

which is discussed in more detail in the following.

1) Conflict Assignment: The update of the map as defined in (19) causes three conflicts

$$
\begin{aligned}
& \zeta\left(\overline{\mathcal{M}}_{t}, \mathcal{M}_{z, t}\right)= \underbrace{m\left(\bar{S}_{t}\right) m\left(F_{z, t}\right)}_{\zeta_{1}}+\underbrace{m\left(\bar{D}_{t}\right) m\left(F_{z, t}\right)}_{\zeta_{2}}+ \\
& \underbrace{m\left(\overline{S D}_{t}\right) m\left(F_{z, t}\right)}_{\zeta_{3}}
\end{aligned}
$$

between predicted occupancy masses and measured free space, which are individually assigned. The static/free conflict $\zeta_{1}$ is equally transferred to $\{S\}$ and $\{F\}$ since both hypotheses are possible and have been measured at the current time or accumulated previously. The dynamic/free conflict $\zeta_{2}$ is assigned to $\{F\}$, as $m\left(F_{z, t}\right)$ describes the latest measurement, whereas $m\left(\bar{D}_{t}\right)$ is only an uncertain prediction. Likewise, the occupancy/free conflict $\zeta_{3}$ is also transferred to the more recent free space measurement since unclassified occupancy $\{S, D\}$ of a previous time, in contrast to $\{S\}$, is also an uncertain state and could be caused by a dynamic object. Hence, all conflicts are assigned to the updated evidence masses of static occupancy or free space:

$$
\begin{aligned}
& m\left(S_{t}\right) \leftarrow \frac{1}{2} \zeta_{1}, \\
& m\left(F_{t}\right) \leftarrow \frac{1}{2} \zeta_{1}+\zeta_{2}+\zeta_{3} .
\end{aligned}
$$

2) Occupancy Derivation: The scan grid $\mathcal{M}_{z, t}$ does not directly measure static or dynamic occupancy; only the superset of unclassified occupancy is measured. This means that the static and dynamic evidence masses

$$
\begin{aligned}
& m\left(S \mid \overline{\mathcal{M}}_{t} \oplus_{\mathrm{c}} \mathcal{M}_{z, t}\right) \leq m\left(\bar{S}_{t}\right), \\
& m\left(D \mid \overline{\mathcal{M}}_{t} \oplus_{\mathrm{c}} \mathcal{M}_{z, t}\right) \leq m\left(\bar{D}_{t}\right), \text { if } m\left(\overline{F D}_{t}\right)=0
\end{aligned}
$$

are not directly increased even if an occupancy measurement $m\left(S D_{z, t}\right)>0$ occurs. Since static or dynamic occupancy are also not derived from $\{S, D\}$ in the prediction, static evidence would never appear in the updated map and dynamic evidence would only be deduced if measured occupancy is combined with accumulated free space of the previous map.

The update of the unclassified occupancy mass

$$
\begin{array}{r}
m\left(S D \mid \overline{\mathcal{M}}_{t} \oplus_{\mathrm{c}} \mathcal{M}_{z, t}\right)=\underbrace{m\left(\overline{S D}_{t}\right) m\left(\Theta_{z, t}\right)}_{\lambda_{1}}+ \\
\underbrace{m\left(\overline{S D}_{t}\right) m\left(S D_{z, t}\right)}_{\lambda_{2}}+\underbrace{m\left(\bar{\Theta}_{t}\right) m\left(S D_{z, t}\right)}_{\lambda_{3}}
\end{array}
$$

using the conjunctive rule of combination (7) has to be modified to derive also static and dynamic occupancy from measured unclassified occupancy. For this purpose, the summands $\lambda_{1}, \lambda_{2}, \lambda_{3}$ in (23) are interpreted differently and individually assigned to $\{S\},\{D\}$, or $\{S, D\}$.
The first term $\lambda_{1}$ depicts the combination of the predicted occupancy mass $m\left(\overline{S D}_{t}\right)$ with the remaining unknown mass of the measurement $m\left(\Theta_{z, t}\right)$, which is defined as

$$
m\left(\Theta_{z, t}\right)=1-m\left(S D_{z, t}\right)-m\left(F_{z, t}\right)
$$

since only $\{S, D\}$ and $\{F\}$ are modeled in the scan grid $\mathcal{M}_{z, t}$. This means that $\lambda_{1}$ equals $m\left(\overline{S D}_{t}\right)$ if no measurement occurs. In such a case, the predicted map $\overline{\mathcal{M}}_{t}$ should not change, i. e.,

$$
m\left(\Theta_{z, t}\right)=1 \Rightarrow \mathcal{M}_{t}=\overline{\mathcal{M}}_{t} .
$$

Hence, the summand $\lambda_{1}$ remains assigned to $\{S, D\}$ :

$$
m\left(S D_{t}\right) \leftarrow \lambda_{1} .
$$

The second term $\lambda_{2}$ represents the mutual support of the occupancy masses of $\overline{\mathcal{M}}_{t}$ and $\mathcal{M}_{z, t}$. An occupied cell should converge toward static occupancy $\{S\}$ when multiple occupancy measurements for that same cell are accumulated. Thus, the static occupancy mass is increased by $\lambda_{2}$ :

$$
m\left(S_{t}\right) \leftarrow \lambda_{2} .
$$

The sum of the first two terms

$$
\lambda_{1}+\lambda_{2}=m\left(\overline{S D}_{t}\right)\left(1-m\left(F_{z, t}\right)\right) \leq m\left(\overline{S D}_{t}\right)
$$

corresponds at most to the predicted occupancy mass $m\left(\overline{S D}_{t}\right)$, i. e., it models the decrease of the predicted occupancy mass by a conflicting free space measurement $m\left(F_{z, t}\right)$. Only the third term $\lambda_{3}$ models the increase of the occupancy mass.

Dynamic occupancy has to be initialized and increased differently than the convergence of static occupancy derived from accumulated occupancy at the same cell. Instead, dynamic occupancy is primarily characterized by newly occurring occupancy. Hence, an amount $f_{D} \in[0,1]$ of the newly increased occupancy mass $\lambda_{3}$ is assigned to $\{D\}$ :

$$
\begin{aligned}
m\left(D_{t}\right) & \leftarrow f_{D} \lambda_{3}, \\
m\left(S D_{t}\right) & \leftarrow\left(1-f_{D}\right) \lambda_{3} .
\end{aligned}
$$

The amount $\left(1-f_{D}\right)$ remains in $\{S, D\}$, whereby $f_{D}<1$ results in slow convergence toward static occupancy with several occupancy measurements of that same cell, cf. (27). The variable $f_{D}$ is modeled as a function of the ratio between the number of predicted particles $\left|\hat{\mathcal{X}}_{t}^{c}\right|$ in that cell and the defined maximum number of particles $n_{\max }$ in a cell. To achieve fast convergence of dynamic occupancy, $f_{D}$ is modeled

$$
f_{D} \equiv \sqrt{\frac{\left|\hat{\mathcal{X}}_{t}^{c}\right|}{n_{\max }}}, \quad\left|\hat{\mathcal{X}}_{t}^{c}\right| \leq n_{\max }
$$

as the square root of this ratio. Dynamic evidence is initialized by spreading particles in cells with unclassified occupancy as discussed in the following Sec. IV-C. These particles initially have no individual dynamic occupancy value, i. e., $o_{\chi}=0$, hence, no predicted dynamic occupancy mass is derived, cf. (14). New dynamic occupancy in (29) is thus only derived when predicted particles are confirmed by an occupancy measurement in that cell.

Moreover, dynamic occupancy is also implicitly increased by the selected set of hypotheses $2^{\Theta}$ together with the modeled prediction. Previously measured free space is transferred to 


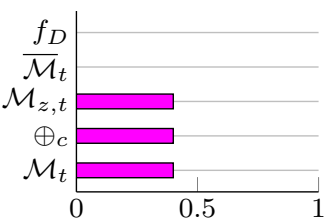

(a)

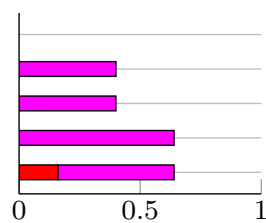

(b)

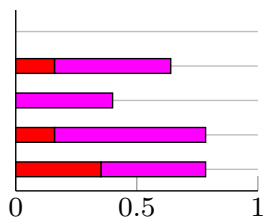

(c)

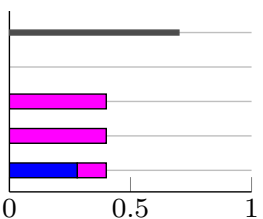

(d)

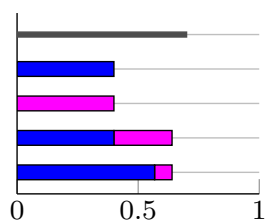

(e)

Fig. 4. Exemplary illustration of occupancy derivation. Rows from top to bottom in all examples: factor $f_{D}$ depending on the number of predicted particles, predicted map $\overline{\mathcal{M}}_{t}$, measured scan grid $\mathcal{M}_{z, t}$, conjunctive combination $\oplus_{c}$ of $\overline{\mathcal{M}}_{t}$ and $\mathcal{M}_{z, t}$, and proposed combination of $\overline{\mathcal{M}}_{t}$ and $\mathcal{M}_{z, t}$ as defined in (33). (a)-(e) show different examples of varied predicted values of $f_{D}$ and $\overline{\mathcal{M}}_{t}$, whereas $m\left(S D_{z, t}\right)=0.4$ is equal in all examples.

$m(F D)$ in the prediction as explained in (15). In such a case, a new measured occupancy $m\left(S D_{z, t}\right)$ results in an increased $m\left(D_{t}\right)$, since the intersection of $\{F, D\}$ and $\{S, D\}$ is $\{D\}$ :

$$
\begin{array}{r}
m\left(D \mid \overline{\mathcal{M}}_{t} \oplus_{\mathrm{c}} \mathcal{M}_{z, t}\right)=m\left(\bar{D}_{t}\right)\left(1-m\left(F_{z, t}\right)\right)+ \\
\underbrace{m\left(\overline{F D}_{t}\right) m\left(S D_{z, t}\right)}_{\lambda_{4}} .
\end{array}
$$

This corresponds to the desired behavior that observed passable area that is currently occupied has to be of a dynamic obstacle. However, completely resolving this toward $\{D\}$ is error-prone to measurement and odometry errors as discussed in Sec. II. Thus, in this work, measured occupancy is partly assigned to $m(S D)$ even though free space was accumulated before. A design parameter $\gamma \in[0,1]$ regulates the modeled uncertainty of the assignment of $\lambda_{4}$

$$
\begin{aligned}
m\left(D_{t}\right) & \leftarrow(1-\gamma) \lambda_{4}+f_{D} \gamma \lambda_{4}, \\
m\left(S D_{t}\right) & \leftarrow\left(1-f_{D}\right) \gamma \lambda_{4},
\end{aligned}
$$

while $\gamma=0$ corresponds to a total assignment of $\lambda_{4}$ to $m\left(D_{t}\right)$ without any modeled uncertainty as in (31). The remaining amount $\gamma \lambda_{4}$ that is not implicitly derived as $\{D\}$ represents an increased unclassified occupancy evidence like the term $\lambda_{3}$. As in (29), a part $f_{D}$ of that is still assigned to $\{D\}$, which, however, depends on the number of predicted particles and not the accumulated evidence mass of passable area $m\left(\overline{F D}_{t}\right)$.

3) Resulting Evidence Masses: The modified mass assignments of the conflicts $\zeta_{1}, \zeta_{2}, \zeta_{3}$ in (21) and the terms $\lambda_{1} \cdot \lambda_{2}, \lambda_{3}$ of the combined occupancy mass in (23) as well as $\lambda_{4}$ of the combined dynamic occupancy mass in (31) finally result in

$$
\begin{aligned}
m\left(S_{t}\right)= & m\left(S \mid \overline{\mathcal{M}}_{t} \oplus_{\mathrm{c}} \mathcal{M}_{z, t}\right)+\frac{1}{2} \zeta_{1}+\lambda_{2}, \\
m\left(D_{t}\right)= & m\left(D \mid \overline{\mathcal{M}}_{t} \oplus_{\mathrm{c}} \mathcal{M}_{z, t}\right)-\left(1-f_{D}\right) \gamma \lambda_{4} \\
& +f_{D} \lambda_{3}, \\
m\left(S D_{t}\right)= & m\left(S D \mid \overline{\mathcal{M}}_{t} \oplus_{\mathrm{c}} \mathcal{M}_{z, t}\right)-\lambda_{2}-f_{D} \lambda_{3} \\
& +\left(1-f_{D}\right) \gamma \lambda_{4}, \\
m\left(F_{t}\right)= & m\left(F \mid \overline{\mathcal{M}}_{t} \oplus_{\mathrm{c}} \mathcal{M}_{z, t}\right)+\frac{1}{2} \zeta_{1}+\zeta_{2}+\zeta_{3}, \\
m\left(F D_{t}\right)= & m\left(F D \mid \overline{\mathcal{M}}_{t} \oplus_{\mathrm{c}} \mathcal{M}_{z, t}\right) .
\end{aligned}
$$

Fig. 4 illustrates the modified occupancy derivation in comparison to the conjunctive combination for different examples.

4) Additional Radar Doppler Measurements: The sensor setup used in this work also includes radar sensors. The occupancy measurement of a grid cell can thus additionally have a radial velocity measurement $v_{z, r}$, which is used to classify dynamic occupancy of unclassified occupancy measurements. Hence, if a radial velocity measurement exists for a cell, the amount $f_{D}$ of newly increased occupancy that is assigned to $\{D\}$ as defined in (30) is adapted to

$$
f_{D}^{v}=\max \left(f_{D}, 1-\exp \left(\frac{-v_{z, r}^{2}}{2 \sigma_{v_{z, r}}^{2}}\right)\right) .
$$

The radial velocity measurement does not decrease the belief that a cell is dynamic since a moving object can still have a radial velocity of zero. However, the dynamic belief increases with an increased radial velocity magnitude $\left|v_{z, r}\right|$, which is modeled by the inverse of an unnormalized zero-mean Gaussian distribution of the measurement $v_{z, r}$ with variance $\sigma_{v_{z, r}}^{2}$.

\section{Computation of New Particle Population}

The new map $\mathcal{M}_{t}$ including the dynamic mass $m\left(D_{t}\right)$ has been updated with the measurement as described above. The particle population is linked to the map and used to predict the dynamic evidence mass as discussed in (14). Consequently, a new particle population $\mathcal{X}_{t}$ has to be computed with respect to the new dynamic mass $m\left(D_{t}\right)$ of the map and the previously predicted particles $\hat{\mathcal{X}}_{t}$ to retain a consistent dynamic mass representation of the map and the particle population.

The new number of particles in a grid cell $c$ is adapted proportionally to the cell density

$$
\rho^{c}=m\left(D_{t}^{c}\right)+\left(1-f_{D}\right)\left(\lambda_{3}+\gamma \lambda_{4}\right)
$$

relative to the maximum number of particles in a cell $n_{\max }$. The density $\rho^{c}$ corresponds to the updated evidence mass of dynamic occupancy and, in addition, the increased unclassified occupancy mass as defined in (33). This means that particles are also initialized with unclassified occupancy, which is required to derive new dynamic occupancy as discussed in (29). The number of the predicted particle population $\left|\hat{\mathcal{X}}_{t}^{c}\right|$ is at most decreased by a factor $\kappa_{p} \in(0,1)$ to prevent an abrupt reduction of the number of particles and hence increase robustness against missing or wrong measurements. In sum, the new number of particles is set to

$$
\left|\mathcal{X}_{t}^{c}\right|=\left\lfloor\max \left(\rho^{c} n_{\max }, \kappa_{p}\left|\hat{\mathcal{X}}_{t}^{c}\right|\right)\right\rfloor .
$$

In the resampling step of the grid-based particle filter, $\left|\mathcal{X}_{t}^{c}\right|$ new particles are drawn from the predicted particle population $\hat{\mathcal{X}}_{t}^{c}$, weighted by radial velocity measurements $v_{z, r}$ if available, with an additional small amount of random particles to reduce particle deprivation as presented in [7]. The new particle occupancy values in a cell are uniformly set to

$$
o_{\chi}=\frac{m\left(D_{t}^{c}\right)}{\left|\mathcal{X}_{t}^{c}\right|}, \quad \chi \in \mathcal{X}_{t}^{c},\left|\mathcal{X}_{t}^{c}\right|>0
$$

such that their sum corresponds to the new dynamic mass $m\left(D_{t}^{c}\right)$ of the updated map $\mathcal{M}_{t}$ as defined in (10). 
In conclusion, particle hypotheses are spread in areas with dynamic occupancy or unclassified occupancy that may be caused by dynamic objects. The actual dynamic mass linked to all particles, however, is defined by the dynamic mass $m\left(D_{t}\right)$ of the map and is consistently filtered.

\section{Occupancy Classification of Scan Grid}

The dynamic grid map $\mathcal{M}_{t}$ represents an accumulated estimation and classification of all hypotheses $\theta \subseteq \Theta$. This filtered estimation is additionally used to distinguish static and dynamic occupancy of the measured occupancy belief $\operatorname{bel}\left(O_{z, t}\right)=m\left(S D_{z, t}\right)$ of the scan grid $\mathcal{M}_{z, t}$. The occupancy belief remains as measured, only the subdivision between $\{S\}$, $\{D\}$, and $\{S, D\}$ is adapted by the filtered map. This results in a pseudo measurement $\tilde{z}$ with the occupancy evidence masses

$$
\begin{aligned}
m\left(S_{\tilde{z}, t}\right) & =m\left(S_{t}\right) m\left(S D_{z, t}\right), \\
m\left(D_{\tilde{z}, t}\right) & =m\left(D_{t}\right) m\left(S D_{z, t}\right), \\
m\left(S D_{\tilde{z}, t}\right) & =m\left(S D_{z, t}\right)-m\left(S_{\tilde{z}, t}\right)-m\left(D_{\tilde{z}, t}\right),
\end{aligned}
$$

compliant with $\operatorname{bel}\left(O_{\tilde{z}, t}\right)=\operatorname{bel}\left(O_{z, t}\right)$, i. e.,

$$
m\left(S_{\tilde{z}, t}\right)+m\left(D_{\tilde{z}, t}\right)+m\left(S D_{\tilde{z}, t}\right)=m\left(S D_{z, t}\right) .
$$

This classified scan grid representation is used as input to the grid-based object tracking [6]. Since that object tracking approach performs a temporal filtering on the object-level, an uncorrelated and currently measured occupancy belief is required as input to avoid multiple filtering of occupied grid cells.

\section{EXPERIMENTAL RESULTS}

The proposed approach has been tested with real sensor data of autonomous driving test vehicles in various urban and highway scenarios. The sensor setup consists of four laser scanners, positioned at the front, front left, front right, and the rear, and four short-range radar sensors installed in the corners. In addition, high-precision inertial measurement units (IMU) are used for odometry estimation. The implementation is based on fast GPU computing parallelized over grid cells and particles. This allows for a real-time application of the overall system that processes measurement data with an input rate of about $25 \mathrm{~Hz}$. All results are generated with a grid size of $680 \times 680$ cells, a cell resolution of $0.2 \mathrm{~m} \times 0.2 \mathrm{~m}$, and a maximum of $n_{\max }=100$ particles per cell. In the following, the successful application of the proposed approach is demonstrated qualitatively for various challenging traffic scenarios. In addition, effects of the modeled uncertainty $\gamma$ as used in the implicit derivation of dynamic occupancy are discussed. Finally, our approach is compared with the approach of [7] including a quantitative evaluation.

\section{A. Qualitative Results}

First, the different processing steps of the overall grid-based environment estimation approach are illustrated and briefly explained to better understand the context of this work. Fig. 5 depicts these steps for a challenging traffic scenario with multiple surrounding vehicles, cyclists, and pedestrians.

Measurement data of lidar and radar sensors, visualized in Fig. 5a, form the input data and enable the environment per-

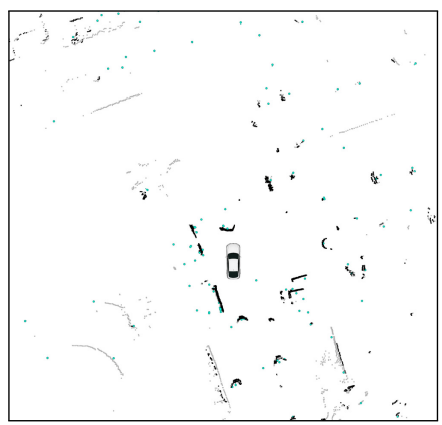

(a)

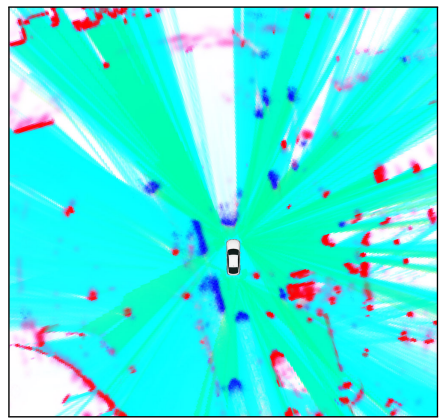

(c)

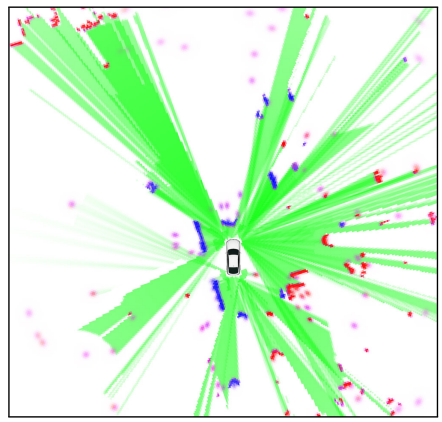

(e)

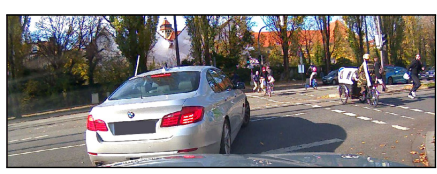

(g)

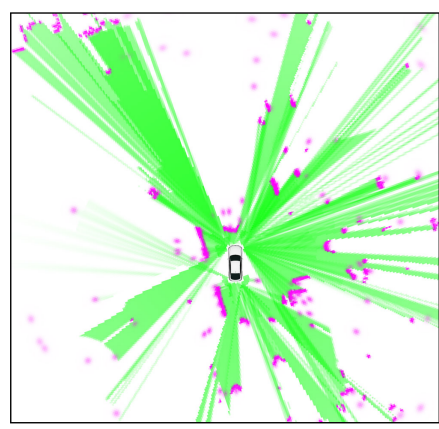

(b)

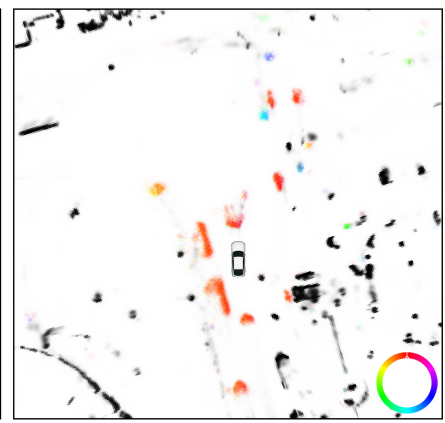

(d)

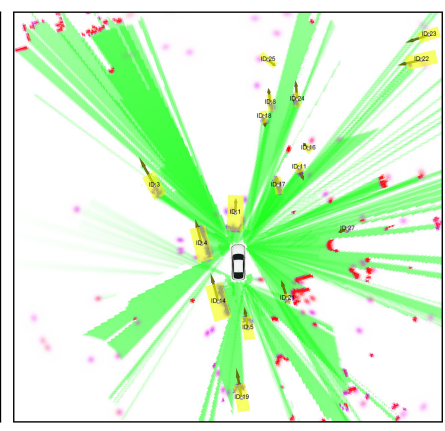

(f)

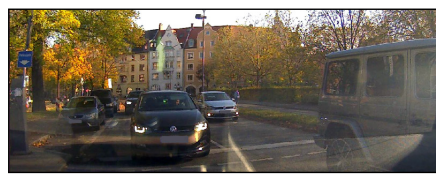

(h)
Fig. 5. Processing steps of overall grid-based environment estimation approach. (a) Sensor measurement data, lidar detections are shown as black (obstacles) and gray (ground points) squares, radar detections are shown as cyan points. (b) Fused scan grid $\mathcal{M}_{z, t}$. (c) Dynamic grid map $\mathcal{M}_{t}$. (d) Velocity orientation grid. (e) Classified scan grid. (f) Grid-based object tracking as proposed in [6]. (g) Front camera image. (h) Rear camera image.

ception. Sensor-individual scan grids with a uniform evidential representation are generated from the detections of each sensor. Since measurements of the used radars have significantly more noise than those of the laser scanners, occupancy of the radar scan grids is modeled with higher uncertainty. All eight sensor scan grids are combined using (9), resulting in a fused scan grid $\mathcal{M}_{z, t}$, shown in Fig. 5b, that forms the input grid of this work. The accumulated odometry, estimated by the integrated IMU, is used as a reference frame of all grids. No rotations and only integer translations (regarding the cell resolution) are performed between different grids to achieve an exact overlap of cells. Hence, the pose of the ego vehicle inside the grid varies over time, e. g., [31], [32]. 


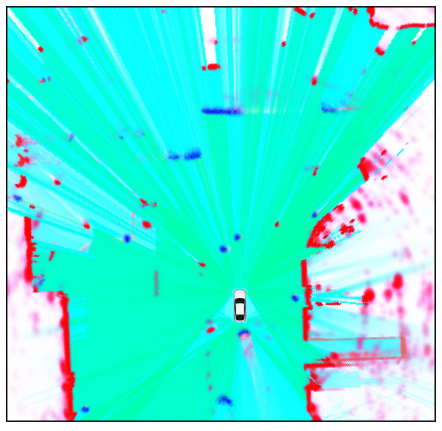

(a)

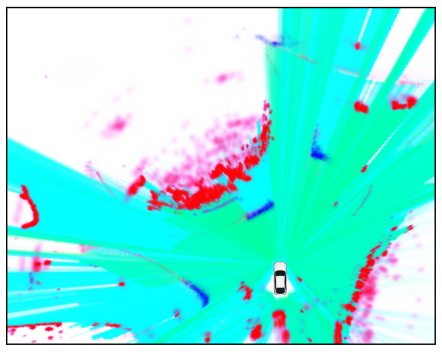

(c)

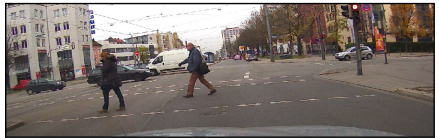

(e)

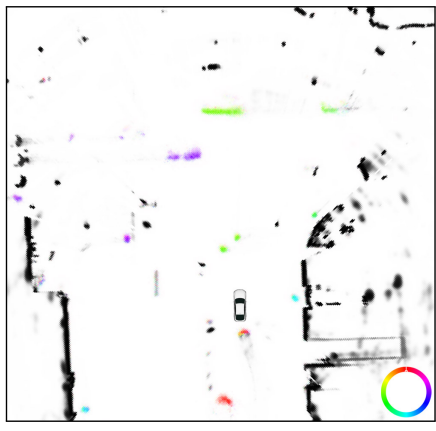

(b)

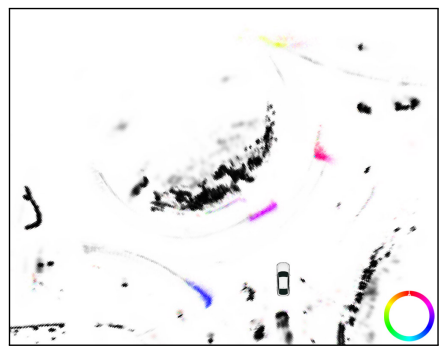

(d)

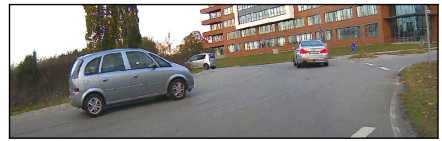

(f)
Fig. 6. Traffic scenarios at a junction (first row) and a roundabout (second row). (a)+(c) Dynamic grid map of first and second scenario, respectively. (b) $+(d)$ Corresponding velocity orientation grids. (d)+(e) Camera images of scenarios of first row (left) and second row (right).

The proposed dynamic grid mapping is presented in Fig. 5c. It represents the filtered and classified estimation of the modeled evidential hypotheses. Static and dynamic occupancy are robustly distinguished without requiring specific object assumptions. The low-level particle tracking estimates cell velocity distributions and initializes and predicts the dynamic occupancy masses. Fig. 5d highlights the estimated cell velocity orientation of the differently moving objects.

The accumulated dynamic grid map $\mathcal{M}_{t}$ is additionally used to distinguish static and dynamic occupancy of the measured occupancy belief $m\left(S D_{z, t}\right)$, cf. (38). The resulting classified scan grid, shown in Fig. 5e, is used to update our grid-based object tracking approach [6]. The resulting tracks using a boxmodel representation are shown in Fig. $5 \mathrm{f}$. In that scenario, all visible 17 moving objects are successfully detected and tracked on both abstraction levels, i. e., the low-level particle tracking and the subsequent high-level object tracking.

Two other challenging scenarios are shown in Fig. 6. The first row shows an intersection with crossing traffic. All detected vehicles and pedestrians are correctly tracked and classified as dynamic occupancy. The second row shows a scenario with four vehicles driving in a roundabout. Again, all objects are classified as dynamic and the moving directions of the turning vehicles are correctly estimated. However, also slight occupancy artifacts behind some objects occur in that scenario. The filter is designed to ensure slow convergence toward static occupancy when a grid cell is measured as unclassified occupied for multiple times. This is also the

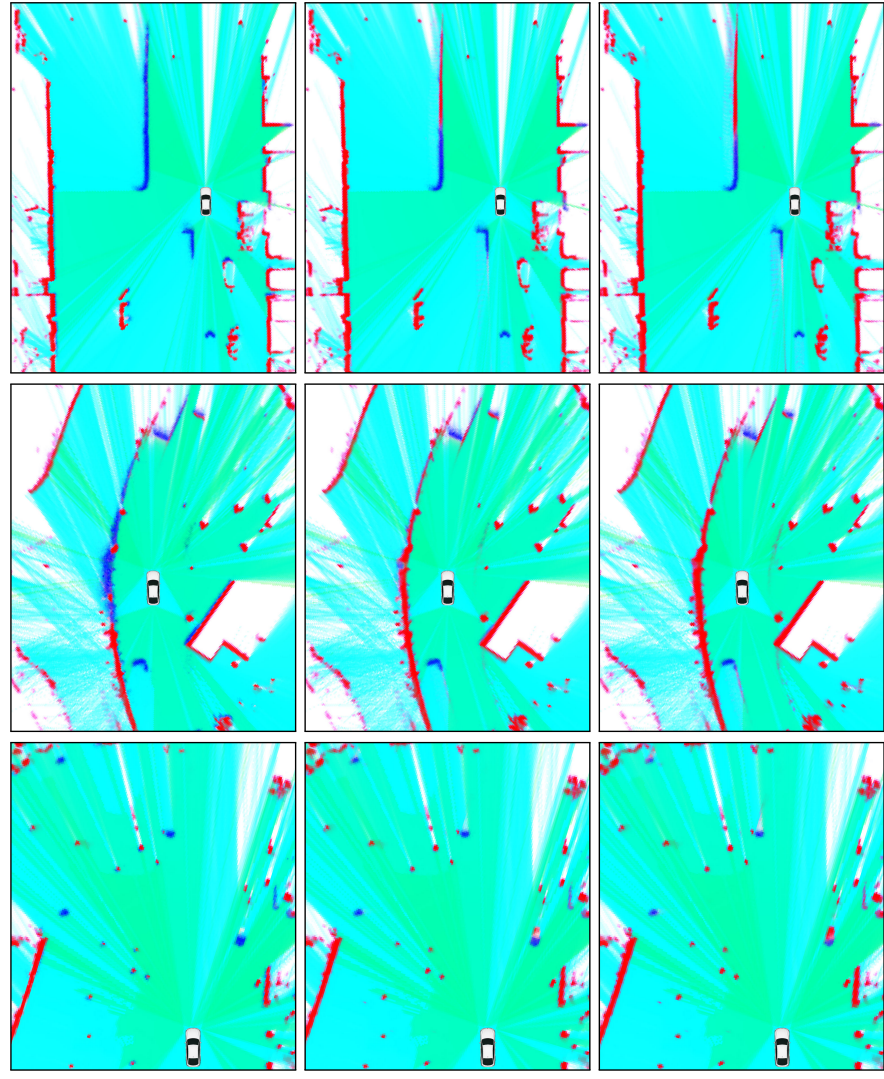

(a) $\gamma=0$.

(b) $\gamma=0.5$

(c) $\gamma=1$
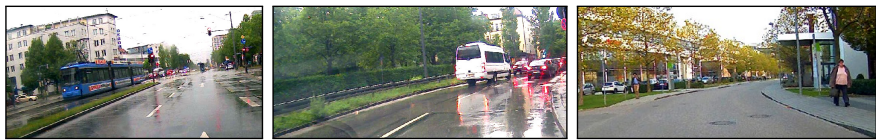

(d) Camera images of scenarios of each row (images from left to right).

Fig. 7. Variation of uncertainty parameter $\gamma$ as used in (32). Input data are exclusively from lidar measurements. Scenario in the first row shows a long dynamic object moving across previously correctly measured free space. The second row shows a static road boundary with previously wrongly measured free space. The third row shows pedestrians moving at different speeds across previously correctly measured free space.

case for cells on the long side of slow-moving objects. The regulation of the convergence between static and dynamic occupancy is discussed in the next section.

\section{B. Parameter Evaluation and Discussion}

The selected frame of discernment $\Theta$, especially the hypothesis $\{F, D\}$ of passable area, allows for the implicit resolution of measured unclassified occupancy $m\left(S D_{z, t}\right)$ toward $\{D\}$ when the map has accumulated $m\left(F D_{t}\right)$ of previously measured free space $m\left(F_{z, t}\right)$. This approach helps to detect moving objects faster, however, it is also error-prone to wrongly derived free space or odometry inaccuracies. Therefore, an uncertainty parameter $\gamma$ has been introduced in (32) to partly transfer an amount of newly increased occupancy toward $m\left(S D_{t}\right)$.

Fig. 7 demonstrates the effects of the modeled uncertainty $\gamma$. The upper row shows an example with a long dynamic object, a tram, moving across previously correctly measured free space. Since $m\left(F D_{t}\right)$ is correct in that case, the result 


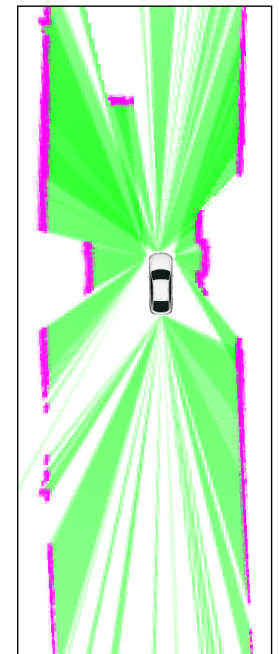

(a)

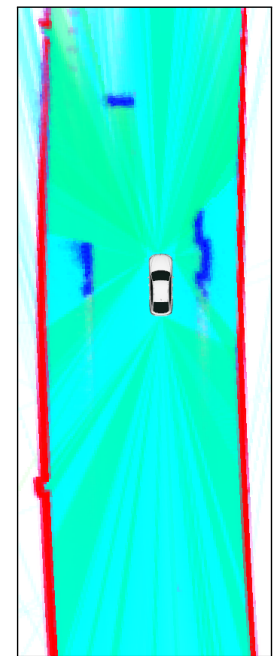

(b)

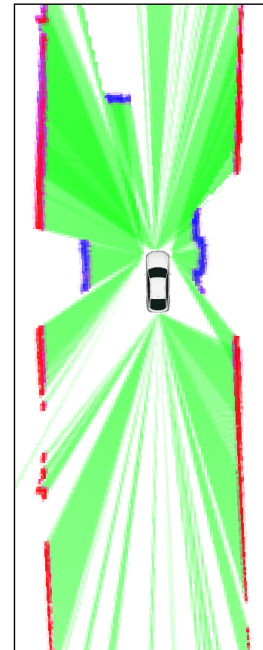

(c)

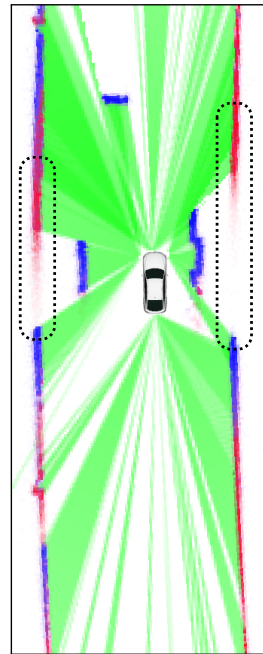

(d)

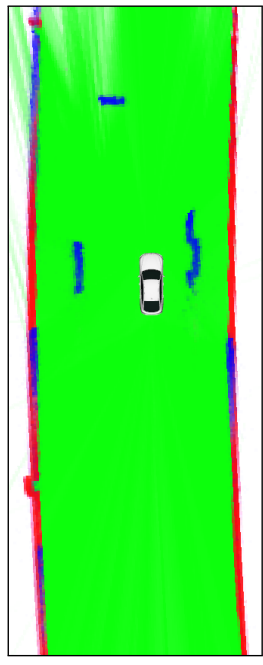

(e)

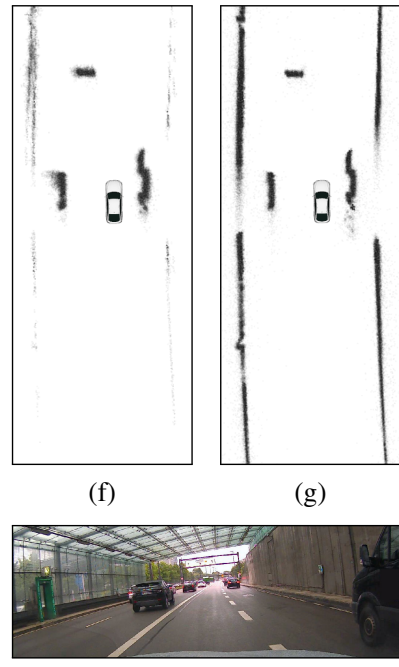

(h)

Fig. 8. Comparison of static/dynamic classification in a scenario with guardrails temporarily occluded by vehicles. The ego vehicle and three vehicles on its left, right, and front left are moving north. Input data are exclusively from lidar measurements. (a) Scan grid. (b) Dynamic map. (c) Classified scan grid. (d) Particle map of [7], not limited to scan grid evidence here to visualize particle destruction. The dotted areas highlight the critical destruction of static particles due to occlusion. (e) DST map of [7]. (f) Particle population of proposed approach. (g) Particle population of [7]. (h) Camera image of scene.

without modeled uncertainty, $\gamma=0$, shows a correct dynamic classification without convergence toward static occupancy. Using $\gamma=1$, no information of previously measured free space is used to derive $\{D\}$, resulting in a fast convergence toward $\{S\}$. Hence, in that scenario, the back part of the tram is wrongly classified as static.

In contrast, the previously measured free space of the second scenario is wrong, resulting in a permanent dynamic classification of the road boundary when no uncertainty is modeled. The introduced uncertainty $\gamma>0$, however, guarantees convergence toward static occupancy even with such wrongly accumulated passable area evidence. The third row shows a scenario with multiple pedestrians moving at different speeds. Here, the measured free space evidence enables a faster convergence toward the dynamic occupancy of a slow-moving pedestrian on the right sidewalk.

In sum, $0<\gamma<1$ represents a tradeoff between a better estimation of dynamic occupancy and an ensured convergence toward static occupancy. In this work, we used $\gamma=0.6$ for all scenarios, i. e., no scenario-specific convergence optimization was performed. This filter design leads to the correct convergence of static obstacles, but also causes a slow convergence of large and slow-moving objects toward static occupancy when no additional information is used. However, those slow cell velocities cannot be completely resolved on the grid cell-level for several consecutive occupancy measurements on that same grid cell, caused by different parts of an object, without measured radial velocities. In such a case, additional information of the high-level object tracking is required to robustly detect the movement of the overall object and suppress convergence of the corresponding cells toward static occupancy. This combination will be addressed in future work.

\section{Comparison with Original Approach}

The proposed approach is an advancement of the GTAM approach [7]. In the following, both approaches are compared in a scenario with multiple fast-moving vehicles that temporarily occlude parts of the static environment. This scenario is evaluated without radar Doppler velocity measurements, i.e., only occupancy measurements of lidar sensors are used as input data to highlight more clearly the different occupancy filtering and classification of both approaches.

Fig. 8 shows the qualitative results of the resulting evidential grids at a fixed time. The scan grid is visualized in Fig. 8a. Both on the left and the right side of the ego-vehicle is a similarly moving vehicle that temporarily occludes parts of the road boundaries. The evidential dynamic map of the proposed approach is shown in Fig. 8b. Static and dynamic occupancy are robustly filtered and correctly distinguished. The occluded area of the road boundary persistently remains statically occupied. Accordingly, the corresponding classified scan grid shows correct results, cf. Fig. 8c.

In contrast, the GTAM particle map in Fig. 8d that represents the classification of the particle population fails in such a scenario and points out a significant systematic drawback of that approach. Although particles at the road boundaries show a slow but correct convergence behavior from wrong dynamic occupancy to static occupancy, the edges of the temporarily occluded road boundaries are classified as incorrect. The correctly filtered static information is lost when static particles are destructed due to occlusion, which then results in a wrong dynamic classification again when the area is not occluded anymore. Although the output of the particle map including static occupancy is accumulated in the DST map in Fig. 8e, which remains when particles are destructed, it is not used as a feedback to the particle filtering.

This behavior has also been observed in urban scenarios where the sensor field of view can change quickly due to the ego motion and obstacles passing closely to the sensors. For example, similar results occur at house walls with densely parked vehicles in front, where gaps between those near 
obstacles cause a fast-changing sensor field of view and thus fast changing occlusions of the areas behind.

Another decisive advantage of the proposed approach is the significantly reduced number of used particles, as the static environment is directly modeled in the map without requiring static particles in contrast to [7], cf. Fig. 8f and 8g. In that exemplary scene, the population of the entire grid consists of 48, 787 particles, whereas the GTAM approach uses 158,719 particles, i.e., more than three times as many particles.

A further advantage of the proposed dynamic map in comparison to the DST map representation is the introduced hypothesis of $\{F, D\}$. Hence, passable area $\{F, D\}$ that may be temporarily occupied and actual current free space $\{F\}$ are modeled separately, which can be used to analyze the current occlusion of a traffic scenario.

Fig. 9 shows the evidential filtering of the scenario of Fig. 8 over time for one fixed cell of the right road boundary. This illustrates in detail the different consequences of the temporary occlusion, here at $t \in[70,91]$, of a static cell caused by the right vehicle as described above. Note that in this work the impact of the measured evidence of a scan grid in the measurement update of the accumulated map is limited to the interval $\left[0, \eta_{z}\right]$ with $\eta_{z} \leq 1$ regulating the maximum amplitude of a measurement. However, the grid visualization of the scan grid, and correspondingly the classified scan grid and the particle map, is referred to the maximum amplitude without considering the actual impact.

The scenario of Fig. 8 is also evaluated quantitatively in terms of the static/dynamic classification and the number of particles. A binary classification

$$
\Lambda= \begin{cases}\{S\}, & \text { if } m\left(S_{t}\right) \geq \psi_{\Lambda} \\ \{D\}, & \text { else }\end{cases}
$$

is performed for all relevant occupied cells of the scan grid, i. e., $m\left(S D_{z, t}\right) \geq m\left(S D_{\Lambda, \min }\right)$. This classification, with a variation of the threshold $\psi_{\Lambda} \in[0,1]$, is evaluated with labeled ground truth data, resulting in a receiver operating characteristic (ROC) curve. Fig. 10a shows the results of the proposed approach in comparison to [7] for a sequence of 250 frames of the scenario of Fig. 8. This evaluation confirms the qualitatively shown results. Especially for scenarios with temporary occlusion of road boundaries, the false dynamic classification is significantly reduced by the proposed architecture in comparison to [7].

The number of required particles $\left|\mathcal{X}_{t}\right|$ of the evaluated sequence is shown in Fig. 10b. At initialization, both approaches create new particles proportional to the sum of measured occupancy, indicated by the pink dotted line. Our approach uses the filtered occupancy evidence of the map, thus the impact of one measurement is limited to the interval $\left[0, \eta_{z}\right]$; here $\eta_{z}=0.4$ is used, as described above. The approach of [7], in contrast, directly initializes particles proportional to the nonlimited measured occupancy evidence. After the initialization phase, the proposed approach significantly reduces the number of particles, as the static environment converges toward $\{S\}$, which is modeled without particles. Since the ego vehicle is moving forward in that scenario, new cells of unclassified occupancy are detected every step. Around $t \approx 100$, the detected (a)

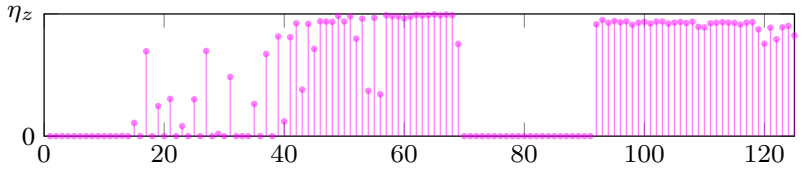

(b)

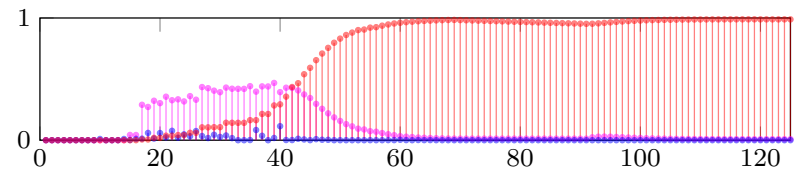

(c)

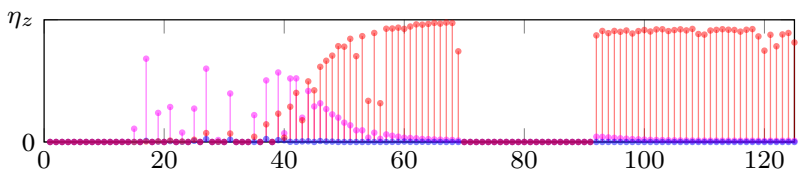

(d)

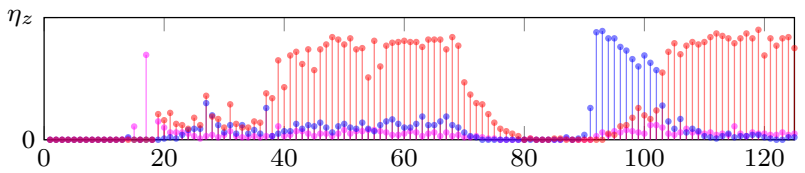

(e)

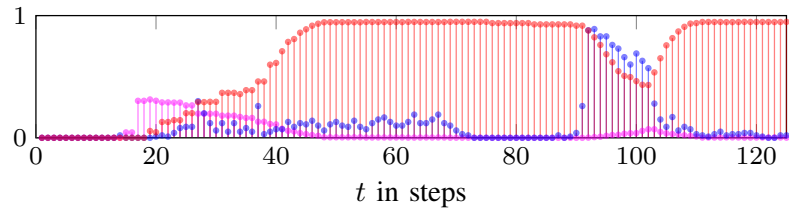

Fig. 9. Temporal filtering of evidence masses at a static cell on the right guardrail of the scenario of Fig. 8. Occupancy masses are visualized as unstacked stems using pink $\widehat{=} m(S D)$, red $\widehat{=} m(S)$, and blue $\widehat{=} m(D)$. The scan grid impact is $\eta_{z}=0.4$. (a) Scan grid. (b) Dynamic map. (c) Classified scan grid. (d) Particle map of [7]. (e) DST map of [7], blue shows non-limited dynamic evidence of particle map without normalization here.

(a)
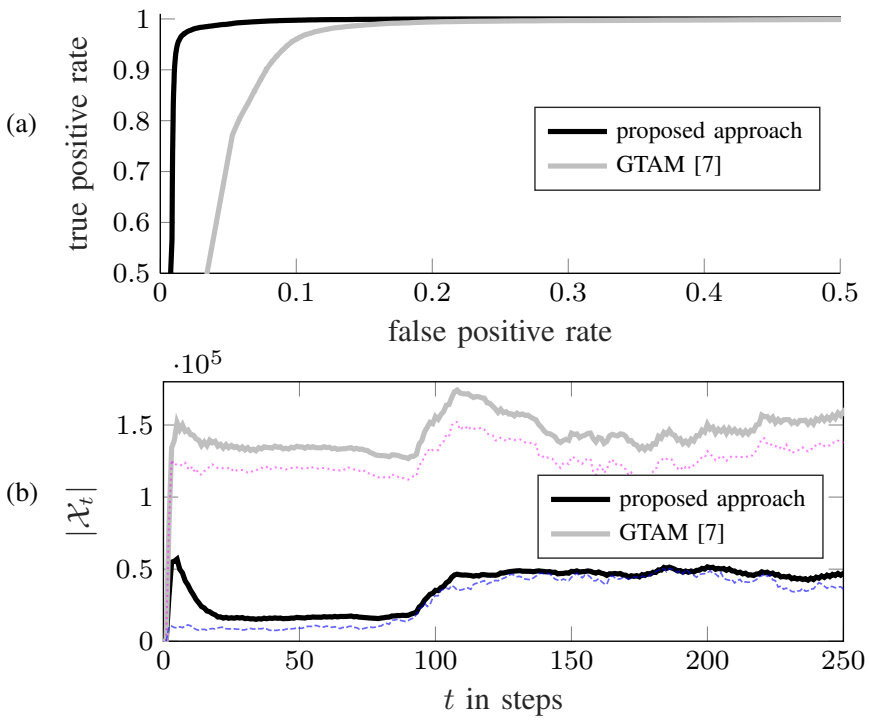

Fig. 10. Quantitative comparison of proposed approach and GTAM [7]. (a) Receiver operating characteristic with a variation of the classification threshold $\psi_{\Lambda}$. Positive denotes the binary classification of dynamic here. (b) Number of total particles $\left|\mathcal{X}_{t}\right|$ with $n_{\max }=100$ particles per cell. Pink dotted line indicates $n_{\max } \sum m\left(S D_{z, t}\right)$ without $\eta_{z}$, blue dashed line indicates number of ground truth dynamic cells of scan grid times $n_{\max }$.

occupied area enlarges significantly, and new dynamic objects occur in the sensor field of view, indicated by the dashed blue line that corresponds to $n_{\max }$ times the number of ground truth dynamic grid cells of the scan grid. Hence, the number of particles increases. The number of particles of [7] remains proportional to the measured occupancy evidence with a large amount of required static particles. 


\section{CONClusions}

This paper has presented a new grid-based estimation approach for dynamic environments in the context of autonomous vehicles. The proposed evidential dynamic grid map represents the fused and accumulated estimation of the local environment, distinguishing between static occupancy, dynamic occupancy, free space, and their combined hypotheses using the Dempster-Shafer framework. Cell velocity distributions are estimated by a low-level particle tracking, enabling the prediction of dynamic evidence masses of the grid map. An adapted evidential filtering has been proposed to combine the map with predicted particles and to update that predicted map with measurement data, including deriving static and dynamic occupancy from measured unclassified occupancy.

Results with real sensor data have shown that the proposed approach robustly distinguishes static obstacles and dynamic objects and correctly estimates cell velocities in various challenging urban and highway scenarios. Compared to a previous approach, especially in scenarios with temporarily occluded areas, false dynamic classification has been significantly reduced by considering the persistent static occupancy accumulation of the grid map. This improved dynamic estimation of grid cells also directly increases the robustness of the subsequent grid-based object tracking by decreasing the number of falsely extracted objects. Furthermore, the computational effort has been significantly reduced since the static environment is directly estimated by the map without requiring static particles. Moreover, it has been shown that using information of previously accumulated measured free space improves the dynamic classification of moving objects, which is implicitly modeled by the combined hypothesis of passable area.

Future work will focus on using object information of the grid-based object tracking within the dynamic grid mapping and the particle tracking to further improve the static/dynamic cell classification, especially to avoid static convergence of cells at long slow-moving objects.

\section{REFERENCES}

[1] K. C. Chang, R. K. Saha, and Y. Bar-Shalom, "On optimal track-totrack fusion," IEEE Trans. Aerosp. Electron. Syst, vol. 33, no. 4, pp. 1271-1276, Oct. 1997.

[2] S. Matzka and R. Altendorfer, "A comparison of track-to-track fusion algorithms for automotive sensor fusion," in Multisensor Fusion and Integration for Intelligent Systems. Springer, 2009, pp. 69-81.

[3] M. Aeberhard, S. Schlichtharle, N. Kaempchen, and T. Bertram, "Trackto-track fusion with asynchronous sensors using information matrix fusion for surround environment perception," IEEE Trans. Intell. Transp. Syst., vol. 13, no. 4, pp. 1717-1726, Dec. 2012.

[4] A. Elfes, "Using occupancy grids for mobile robot perception and navigation," Computer, vol. 22, no. 6, pp. 46-57, Jun. 1989.

[5] S. Thrun, W. Burgard, and D. Fox, Probabilistic Robotics (Intelligent Robotics and Autonomous Agents). The MIT Press, 2005.

[6] S. Steyer, G. Tanzmeister, and D. Wollherr, "Object tracking based on evidential dynamic occupancy grids in urban environments," in Proc. IEEE Intell. Veh. Symp., 2017, pp. 1064-1070.

[7] G. Tanzmeister and D. Wollherr, "Evidential grid-based tracking and mapping," IEEE Trans. Intell. Transp. Syst., vol. 18, no. 6, pp. 1454 1467, June 2017.

[8] C.-C. Wang and C. Thorpe, "Simultaneous localization and mapping with detection and tracking of moving objects," in Proc. IEEE Int. Conf. Robot. Autom., vol. 3, 2002, pp. 2918-2924.
[9] T.-D. Vu, O. Aycard, and N. Appenrodt, "Online localization and mapping with moving object tracking in dynamic outdoor environments," in Proc. IEEE Intell. Veh. Symp., 2007, pp. 190-195.

[10] J. Moras, V. Cherfaoui, and P. Bonnifait, "Credibilist occupancy grids for vehicle perception in dynamic environments," in Proc. IEEE Int. Conf. Robot. Autom., May 2011, pp. 84-89.

[11] R. Jungnickel and F. Korf, "Object tracking and dynamic estimation on evidential grids," in Proc. IEEE Intell. Transp. Syst. Conf., 2014, pp. 2310-2316.

[12] M. Kurdej, J. Moras, V. Cherfaoui, and P. Bonnifait, "Map-Aided Evidential Grids for Driving Scene Understanding," IEEE Intell. Transp. Syst. Mag., vol. 7, no. 1, pp. 30-41, 2015.

[13] T. D. Vu and O. Aycard, "Laser-based detection and tracking moving objects using data-driven Markov chain Monte Carlo," in Proc. IEEE Int. Conf. Robot. Autom., 2009, pp. 3800-3806.

[14] M. Bouzouraa and U. Hofmann, "Fusion of occupancy grid mapping and model based object tracking for driver assistance systems using laser and radar sensors," in Proc. IEEE Intell. Veh. Symp., 2010, pp. 294-300.

[15] M. Schreier, V. Willert, and J. Adamy, "Grid mapping in dynamic road environments: Classification of dynamic cell hypothesis via tracking," in Proc. IEEE Int. Conf. Robot. Autom., 2014, pp. 3995-4002.

[16] R. O. Chavez-Garcia and O. Aycard, "Multiple Sensor Fusion and Classification for Moving Object Detection and Tracking," IEEE Trans. Intell. Transp. Syst., vol. 17, no. 2, pp. 525-534, Feb. 2016.

[17] C. Coué, C. Pradalier, C. Laugier, T. Fraichard, and P. Bessiere, "Bayesian Occupancy Filtering for Multitarget Tracking: an Automotive Application," Intl. J. of Robotics Research, vol. 25, no. 1, pp. 19-30, Jan. 2006.

[18] M. K. Tay, K. Mekhnacha, M. Yguel, C. Coue, C. Pradalier, C. Laugier, T. Fraichard, and P. Bessiere, "The Bayesian occupation filter," in Probabilistic Reasoning and Decision Making in Sensory-Motor Systems. Springer, 2008, pp. 77-98.

[19] M. Saval-Calvo, L. Medina-Valdés, J. M. Castillo-Secilla, S. CuencaAsensi, A. Martínez-Álvarez, and J. Villagrá, "A Review of the Bayesian Occupancy Filter," Sensors, vol. 17, no. 2, p. 344, 2017.

[20] R. Danescu, F. Oniga, and S. Nedevschi, "Modeling and tracking the driving environment with a particle-based occupancy grid," IEEE Trans. Intell. Transp. Syst., vol. 12, no. 4, pp. 1331-1342, Dec. 2011.

[21] R. Danescu, C. Pantilie, F. Oniga, and S. Nedevschi, "Particle grid tracking system stereovision based obstacle perception in driving environments," IEEE Intell. Transp. Syst. Mag., vol. 4, no. 1, pp. 6-20, 2012.

[22] G. Tanzmeister, J. Thomas, D. Wollherr, and M. Buss, "Grid-based mapping and tracking in dynamic environments using a uniform evidential environment representation," in Proc. IEEE Int. Conf. Robot. Autom., 2014, pp. 6090-6095.

[23] A. Nègre, L. Rummelhard, and C. Laugier, "Hybrid sampling bayesian occupancy filter," in Proc. IEEE Intell. Veh. Symp., 2014, pp. 1307-1312.

[24] L. Rummelhard, A. Nègre, and C. Laugier, "Conditional monte carlo dense occupancy tracker," in Proc. IEEE Intell. Transp. Syst. Conf., 2015, pp. 2485-2490.

[25] D. Nuss, T. Yuan, G. Krehl, M. Stuebler, S. Reuter, and K. Dietmayer, "Fusion of laser and radar sensor data with a sequential monte carlo bayesian occupancy filter," in Proc. IEEE Intell. Veh. Symp., 2015, pp. 1074-1081.

[26] D. Nuss, S. Reuter, M. Thom, T. Yuan, G. Krehl, M. Maile, A. Gern, and K. Dietmayer, "A random finite set approach for dynamic occupancy grid maps with real-time application," arXiv preprint arXiv:1605.02406, 2016. [Online]. Available: https://arxiv.org/abs/1605.02406

[27] A. P. Dempster, "Upper and lower probabilities induced by a multivalued mapping," Ann. Math. Statist., vol. 38, no. 2, pp. 325-339, April 1967.

[28] G. Shafer, A Mathematical Theory of Evidence. Princeton University Press, 1976.

[29] A. P. Dempster, “A generalization of bayesian inference," J. Roy. Statist. Soc. Ser. B (Methodol.), vol. 30, no. 2, pp. 205-247, 1968.

[30] E. Lefevre, O. Colot, and P. Vannoorenberghe, "Belief function combination and conflict management," Information fusion, vol. 3, no. 2, pp. 149-162, 2002.

[31] G. Tanzmeister, "Grid-based environment estimation for local autonomous vehicle navigation," Dissertation, Technische Universität München, München, 2016.

[32] K. C. J. Dietmayer, S. Reuter, and D. Nuss, "Representation of Fused Environment Data," in Handbook of Driver Assistance Systems: Basic Information, Components and Systems for Active Safety and Comfort, H. Winner, S. Hakuli, F. Lotz, and C. Singer, Eds. Cham: Springer International Publishing, 2016, pp. 567-603. 


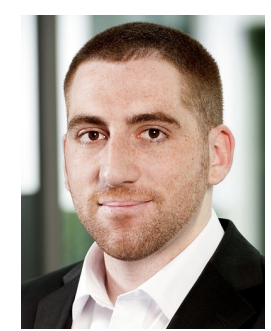

Sascha Steyer received the B.Sc. and M.Sc. degrees in electrical engineering and information technology from Karlsruhe Institute of Technology (KIT), Karlsruhe, Germany, in 2012 and 2015, respectively. He is currently working toward his Dr.-Ing. degree at Technical University of Munich, Munich, Germany, in conjunction with the BMW Group. His research interests include autonomous vehicles, sensor data fusion, and tracking, with a focus on the grid-based estimation of dynamic environments.

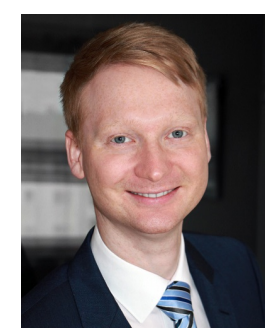

Georg Tanzmeister received the B.Sc. degree in computer science in 2009 from Technische Universität Wien, Vienna, Austria; the Dipl.-Ing. degree in computer graphics and computer vision in 2011 from Technische Universität Wien, Vienna, Austria; and the Dr.-Ing. degree in electrical engineering in 2016 from Technische Universität München, Munich, Germany. Since 2012, he has been Research Engineer for automated driving with the BMW Group. His research interests include autonomous vehicles, gridbased environment estimation, sensor data fusion, and motion planning. In 2016, he received the dissertation award from VDI München and the dissertation award from VDE Südbayern.

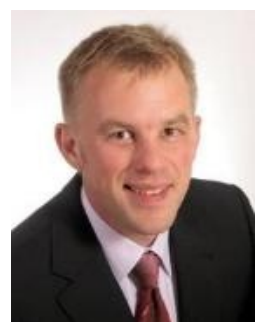

Dirk Wollherr received the Dipl.-Ing. degree in electrical engineering in 2000, the Dr.-Ing. degree in electrical engineering in 2005, and the Habilitation degree in 2013 from Technische Universität München, Munich, Germany. From 2001 to 2004, he was a Research Assistant with the Control Systems Group, Technische Universität Berlin, Germany. In 2004, he was a Research Fellowship with the Japanese Society for the Promotion of Science, Yoshihiko-Nakamura-Lab, The University of Tokyo, Japan. From 2006 to 2008, he was the General Manager of the Cluster of Excellence Cognition for Technical Systems (CoTeSys). Since 2005, he has been a Principal Investigator, the Independent Junior Research Group Leader, and a Research Area Leader with CoTeSys. He was a TUM Carl-von-Linde Junior Fellow with the Institute of Advanced Studies from 2010 to 2013. He has been active in dissemination, such as the General Chair of the German Robotik 2008 Conference, and the Finance Chair of RO-MAN 2008, the Program Co-Chair of the Workshop on Advanced Robotics and its Social Impacts (ARSO) 2013, and also in several EU projects, such as Robot@CWE, CyberWalk, Movement, and IURO. His research interests include automatic control, robotics, autonomous mobile robots, human-robot-interaction, and humanoid walking. 